\title{
Hiper-homocisteinemia e risco cardiometabólico
}

\author{
Hyperhomocysteinemia and cardiometabolic risk
}

Helio Vannucchi', Sandra Soares Melo²

\section{RESUMO}

A hiper-homocisteinemia, quando considerada como fator causal de doenças vasculares, tem suscitado muitas discussões. Estudos caso-controle, retrospectivos e prospectivos têm identifi-

'Divisão de Nutrologia, Faculdade de Medicina de Ribeirão Preto, Universidade de São Paulo (USP), Ribeirão Preto, SP, Brasil

${ }^{2}$ Universidade do Vale do Itajaí (Univali), Itajaí, SC, Brasil; Universidade Gama Filho (UGF), São Paulo, SP, Brasil
Correspondência para:

Helio Vannucchi

Faculdade de Medicina de Ribeirão Preto, USP

Av. Bandeirantes, 3.900

14049-900 - Ribeirão Preto, SP,

Brasil

hvannucc@fmrp.usp.br

Recebido em 25/Mar/2009

Aceito em 28/Mai/2009 cado relação entre concentrações plasmáticas elevadas de homocisteína e doenças vasculares. Na presente revisão, objetivou-se compreender melhor a inter-relação entre as concentrações plasmáticas de homocisteína e doenças vasculares, além do envolvimento de fatores de risco clássicos para a doença: os genéticos, como as mutações em genes que codificam as enzimas envolvidas no metabolismo da homocisteína, e os nutricionais, como a deficiência de vitaminas do complexo B. Foram consultadas as publicações das principais bases de dados em saúde, no período de 1962 a 2009. O mecanismo pelo qual a hiper-homocisteinemia atua como fator de risco para doenças vasculares ainda não está totalmente esclarecido; entretanto, sugere-se o envolvimento da disfunção endotelial e da peroxidação lipídica. 0 tratamento da hiper-homocisteinemia fundamenta-se na suplementação alimentar e medicamentosa, com ácido fólico e vitaminas B6 e B12. Arq Bras Endocrinol Metab. 2009;53(5):540-9.

Descritores

Hiper-homocisteinemia; doenças vasculares; endotélio; peroxidação de lipídeos; mutação; deficiência de vitaminas do complexo B

\begin{abstract}
Hyperhomocysteinemia, when considered as a causal factor of vascular diseases, has been subject of much discussion. Case-control, retrospective and prospective studies have identified a relationship between high plasma concentrations of homocysteine and vascular disease. The aim of the present review was to better understand the interrelation between plasma concentrations of homocysteine and vascular diseases, as well as the involvement of classical risk factors for the disease: genetic factors, such as mutations in the genes that codify the enzymes involved in the metabolism of homocysteine, and nutritional factors, such as complex B vitamin deficiency. The publications of the main databases in health were consulted for the period 1962 to 2009. The mechanism by which hyperhomocysteinemia acts as a risk factor for vascular diseases still has not been fully clarified, but involvement of endothelial dysfunction and lipid peroxidation is suggested. The treatment of hyperhomocysteinemia is based on food supplements and medication, with folic acid and vitamins B6 and B12. Arq Bras Endocrinol Metab. 2009;53(5):540-9.

Keywords

Hyperhomocysteinemia; vascular diseases; endothelium; lipid peroxidation; mutation; vitamin B deficiency
\end{abstract}

\section{INTRODUÇÃO}

A s doenças vasculares são a principal causa de morte em países ocidentais, sendo responsáveis, no Brasil, por cerca de $30 \%$ dos óbitos e por $16 \%$ dos gastos do Sistema Único de Saúde (SUS) (1). Segundo dados de atestados de óbito, em 2005, no Brasil, entre as doenças cardiovasculares, o acidente vascular cerebral foi a pri- meira causa de morte, seguido da doença isquêmica do coração $(1,2)$.

Fatores de risco clássicos para as doenças vasculares, como hipercolesterolemia, hipertensão arterial sistêmica, diabetes melito, tabagismo, obesidade, sedentarismo e antecedentes familiares, são responsáveis por dois terços das causas de mortes $(2,3)$. Entretanto, ressalta-se o fato de $30 \%$ a $35 \%$ dos indivíduos com doenças car- 
diovasculares apresentarem normocolesterolemia, e o de mais de $40 \%$ dos pacientes com doença primária da artéria coronária, cerebrovascular ou vascular periférica terem concentrações plasmáticas de homocisteína elevadas - denominada hiper-homocisteinemia $(3,4)$.

A homocisteína é um aminoácido sulfurado produzido intercelularmente pela desmetilação da metionina. Evidências epidemiológicas mostram que a hiperhomocisteinemia é um fator de risco independente para a doença vascular - doença cerebral, coronariana e arterial periférica (5-8), além de poder contribuir para a manifestação prematura $(9,10)$ e para a severidade da doença arterial coronariana $(11,12)$ e de representar um preditor de mortalidade, independentemente dos fatores de risco tradicionais conhecidos (13).

Embora a hiper-homocisteinemia esteja associada à doença vascular, permanece incerto o quanto a homocisteína representa um fator de risco causal modificável ou forte marcador dessa doença $(14,15)$.

A concentração plasmática de homocisteína é influenciada tanto por fatores nutricionais, tais como o status do ácido fólico e as vitaminas B6 e B12, quanto por fatores hereditários, especialmente ligados às enzimas do metabolismo da metionina e da cisteína, como a metionina sintetase (MS), 5,10 metilenotetrahidrofolato redutase (MTHFR) e cistationina $\beta$-sintetase (CßS), além de estados patológi$\cos$, como a redução da função renal $(16,17)$.

Várias enzimas são envolvidas nas duas vias interconectadas do metabolismo da homocisteína, mas as enzimas MTHFR e C $\beta S$ são as mais extensivamente estudadas. Polimorfismos ou mutações em genes que codificam essas enzimas envolvendo a presença de alelos mutantes (genótipo homozigoto e, em menor extensão, genótipo heterozigoto) podem ser importantes na determinação das concentrações plasmáticas de homocisteína em populações de alto risco para o desenvolvimento de doenças vasculares (18).

Indivíduos homozigotos mutantes com variante termolábil, resultante da transição de $\mathrm{C}$ para $\mathrm{T}$ no códon 677 no gene que codifica a enzima MTHFR, apresentam diminuição na atividade dessa enzima e elevação moderada das concentrações plasmáticas de homocisteína (19). Entretanto, existem resultados contraditórios na literatura com referência ao papel das mutações no gene da MTHFR como fatores de risco independentes para as doenças vasculares (20). O genótipo homozigoto para a mutação 677TT está comumente associado ao risco aumentado de doença arterial coronariana em Israel, na América do Norte e no Japão, mas não em outras populações, como a chinesa, que carrega o mesmo polimorfismo para MTHFR $(17,21)$. A mutação parece ser neutra em relação à concentração plasmática de homocisteína, quando o ácido fólico encontra-se em quantidades adequadas no organismo, mas, naqueles indivíduos com baixa concentração de ácido fólico, a homocisteína aumenta de forma acentuada nos indivíduos homozigotos 677TT e, em valores intermediários, nos heterozigotos. A região na qual se estabeleceu a mutação na enzima tem envolvimento com o ácido fólico e, quando as concentrações dessa vitamina estão adequadas, a enzima pode ser estabilizada (22).

A maioria dos estudos prospectivos apoia o conceito de ser a hiper-homocisteinemia um fator de risco para a doença cardiovascular; metanálises mostraram resultados similares $(23,24)$.

Neste artigo, realizou-se levantamento em bases de dados da literatura científica internacional (MedLine, de 1962 a 2009) e latino-americana (Lilacs, de 1982 a 2009), com busca manual de citações nos respectivos artigos recuperados. Para a pesquisa, foram utilizados descritores da área de Saúde (DeCS) cadastrados na Biblioteca Regional de Medicina (Bireme) do Centro Latino-americano e do Caribe de Informação em Ciências da Saúde: "homocisteína", "doenças vasculares", "doenças cardiovasculares", "fatores de risco", "mutação", "deficiência de vitamina do complexo B" e "vitaminas hidrossolúveis".

Na presente revisão, objetivou-se compreender melhor a inter-relação entre as concentrações plasmáticas de homocisteína e doenças vasculares, além do envolvimento de fatores de risco clássicos para a doença: os genéticos, como as mutações em genes que codificam as enzimas envolvidas no metabolismo da homocisteína, e os nutricionais, como a deficiência de vitaminas B6, B12 e ácido fólico.

\section{CONCEITO E VIAS METABÓLICAS}

A homocisteína é um aminoácido sulfurado formado exclusivamente a partir da desmetilação da metionina proveniente da dieta ou de seu catabolismo (25).

Após sua formação, seu metabolismo poderá seguir por duas vias: a remetilação e a transulfuração (Figura 1). Na remetilação, a homocisteína adquire um grupo metil da 5-metiltetra-hidrofolato ou da betaína para formar a metionina. A reação com 5-metiltetra-hidrofolato acontece em todos os tecidos e tem como cofator a vitamina B12, enquanto a reação com betaína ocorre principalmente no fígado e rins. Na via de transulfuração, 


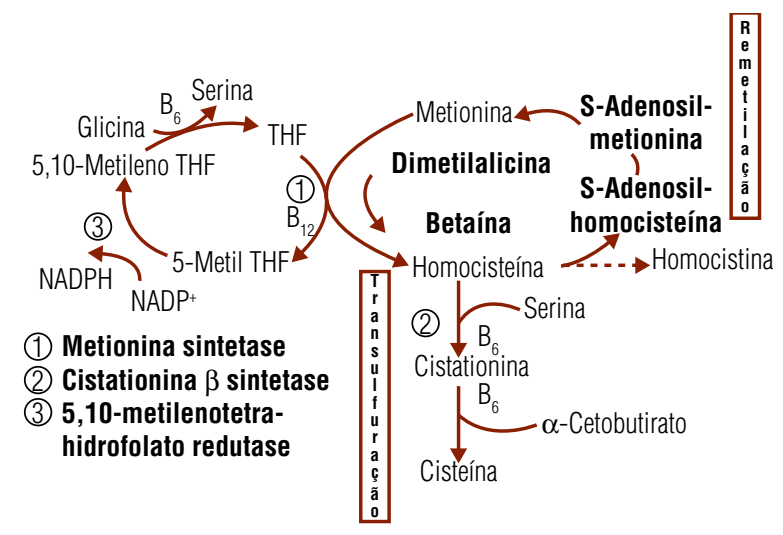

Figura 1. Vias metabólicas da homocisteína.

a homocisteína se condensa com a serina para formar cistationina por meio de uma reação irreversível, dependente de piridoxal fosfato (vitamina B6), formando, ao final, a cisteína $(26,27)$.

A homocisteína plasmática livre é encontrada na forma oxidada, formando dissulfetos que contêm enxofre e que incluem homocistina (dímero da homocisteína) e dissulfetos mistos, como homocisteína-cisteína. Entre 2\% e 5\% da homocisteína plasmática livre estão presentes em sua forma reduzida, e $70 \%$ a $80 \%$ circulam ligados a proteínas plasmáticas, principalmente à albumina. A homocisteína plasmática total é a soma de todas as formas livres e ligadas a proteínas que contenham um grupamento tiol (3).

A homocisteína é liberada para o plasma, a partir dos tecidos, em quantidades reduzidas. Até mesmo quando há um excesso de metionina, o excedente de homocisteína é direcionado para a via de transulfuração. Todos esses mecanismos, de alguma forma, controlam a concentração plasmática. Porém, quando o balanço entre a síntese e o turnover da homocisteína é interrompido, a homocisteína e seus derivados acumulam nas células e no sangue (28).

\section{EVOLUÇÃO HISTÓRICA}

A homocisteína foi descoberta em 1952 (29), porém, só em 1962, foram descritos dois casos de homocistinúria (excreção de metabólitos da homocisteína na urina) (30). Dois anos depois, a homocisteína foi determinada na urina de crianças portadoras de deficiência mental (31). Em 1969, descreveu-se pela primeira vez a relação entre hiper-homocisteinemia, aterosclerose e trombose arterial e venosa em portadores de homocistinúria (32). Entretanto, somente em 1978, a homocisteína foi determinada pela primeira vez em indivíduo saudável
(33), e, em 1985, foi descrita a correlação entre doença vascular e hiper-homocisteinemia em indivíduos heterozigotos para a homocistinúria, com deficiência da enzima C $\beta S$ (34).

\section{CLASSIFICAÇÃ0, MANIFESTAÇÕES CLÍNICAS E PREVALÊNCIA DA HIPER-HOMOCISTEINEMIA}

A determinação bioquímica da homocisteína total permite a identificação de várias formas de hiper-homocisteinemia $(35,36)$. Esta pode ser classificada nas formas moderada, intermediária e grave, de acordo com as concentrações de homocisteína plasmática total entre $14 \mathrm{e}$ 30 , entre 31 e 100 e acima de $100 \mu \mathrm{mol} / \mathrm{L}$, respectivamente (19). O valor de referência para homocisteína plasmática estratificado por sexo é de 6 a $12 \mu \mathrm{mol} / \mathrm{L}$, para mulheres, e 8 a $14 \mu \mathrm{mol} / \mathrm{L}$, para homens $(35,36)$.

Geralmente a hiper-homocisteinemia grave está associada à presença de homocistinúria. A homocistinúria, definida como erro inato do metabolismo de transmissão autossômica recessiva, cuja principal causa é a deficiência de uma ou mais enzimas envolvidas no metabolismo da homocisteína, tem sido considerada importante fator na etiopatogenia da doença vascular, principalmente de grandes vasos (3).

As manifestações clínicas da hiper-homocisteinemia grave incluem anormalidades neurológicas, retardo mental, episódios recorrentes de tromboembolismo e doenças vasculares em idade precoce. Concentrações plasmáticas elevadas de homocisteína estão associadas a defeitos congênitos do tubo neural, como também a defeitos congênitos do coração, abortos e outras complicações da gravidez (19).

A prevalência de hiper-homocisteinemia ocorre em $5 \%$ a $7 \%$ da população geral, porém, concentrações moderadas a intermediárias ocorrem em 13\% a 47\% daqueles com doença vascular aterosclerótica sintomática ou diabetes melito $(22,36)$.

\section{MECANISMOS PLAUSÍVEIS DA AÇÃO DA HOMOCISTEÍNA NA DOENÇA VASCULAR}

Os mecanismos exatos pelos quais a hiper-homocisteinemia favorece o desenvolvimento de doenças vasculares não são totalmente compreendidos (37).

A patogenia da lesão vascular determinada pela hiper-homocisteinemia inclui lesão da célula endotelial, crescimento da musculatura lisa vascular, maior adesividade plaquetária, aumento da oxidação do LDL-coles- 
terol com deposição na parede vascular e ativação direta da cascata da coagulação $(38,39)$.

A hiper-homocisteinemia parece causar principalmente alterações do endotélio vascular, mediadas pelo efeito tóxico-oxidativo da homocisteína. No plasma, parte da homocisteína é auto-oxidada, formando superóxidos e peróxido de hidrogênio, o que poderia causar lesão da célula endotelial, ativação plaquetária e trombose (38).

Dessa forma, foi proposto que a homocisteína causa dano endotelial grave e que esta injúria conduz à ativação plaquetária, à proliferação de células musculares lisas e à trombose. Além disso, a homocisteína pode induzir a atenuação da biodisponibilidade do óxido nítrico, causando a diminuição das propriedades antitrombóticas do endotélio e ocasionando a ativação plaquetária e a geração de trombina (40).

$\mathrm{O}$ aumento na produção das espécies reativas de oxigênio poderia também estar envolvido no dano ao DNA induzido pela homocisteína. A homocisteína induz o dano de DNA e a apoptose celular mediados pela elevação da geração intracelular de peróxido de hidrogênio. A necrose e a instabilidade genética de linfócitos humanos são induzidas pelo peróxido de hidrogênio. Assim, sugere-se que, em concentrações elevadas, a homocisteína desempenhe papel genotóxico (41).

Há ainda muito a ser estudado sobre a influência da homocisteína na função das células sanguíneas e do endotélio vascular, principalmente se a homocisteína for um fator de risco ou uma consequência da doença vascular.

\section{HIPER-HOMOCISTEINEMIA E DOENÇAS VASCULARES}

A elevação moderada de homocisteína no plasma como fator causal de doenças vasculares tem motivado muitas discussões. Alguns estudos caso-controle (42), retrospectivos (43) e prospectivos (44) têm identificado relação entre concentrações elevadas de homocisteína e doenças vasculares de início recente em artérias coronárias, cerebrais e periféricas. Detectou-se hiper-homocisteinemia em $42 \%$ dos pacientes com doença cerebrovascular, em $28 \%$ com doença vascular periférica e em $30 \%$ com doença vascular coronariana (45).

Wald e cols. (46), na tentativa de esclarecer se era causal a associação da hiper-homocisteinemia à doença isquêmica do coração, à trombose e ao derrame, realizaram metanálise com 72 estudos, nos quais a prevalência da mutação no gene da MTHFR foi anali- sada em casos e controles $(\mathrm{n}=16.849)$, e em mais 20 estudos prospectivos, que examinaram a relação entre homocisteína sérica e risco de doença $(\mathrm{n}=3.820)$. Os autores encontraram associação significativa entre a homocisteína e as três doenças estudadas. Estudos genéticos e estudos prospectivos não compartilharam a mesma fonte potencial de erro, e o fato de terem produzido resultados similares reforça a hipótese de que a associação entre homocisteína e doença vascular deve ser causal.

Metanálise envolvendo 27 trabalhos e mais de 4 mil pacientes concluiu que, quando os valores de homocisteína eram maiores que $10 \mu \mathrm{mol} / \mathrm{L}$, incrementos de $5 \mu \mathrm{mol} / \mathrm{L}$ nos valores de homocisteína circulante associavam-se a $80 \%$ de risco para doença cardiovascular em mulheres e $60 \%$ em homens, e $50 \%$ para doença cerebrovascular, além de aumentar em 6,8 vezes o risco para doença vascular periférica (47).

May e cols. (48) avaliaram o quanto o aumento da homocisteína prediz falência cardíaca congestiva. Foram estudados 2.842 pacientes com angiografia coronariana. Disfunção ventricular esquerda e falência cardíaca congestiva foram confirmadas respectivamente em $12 \%$ e $21,9 \%$ dos pacientes com concentrações plasmáticas de homocisteína $\geq 13,3 \mu \mathrm{mol} / \mathrm{L}$. Após ajustes, a homocisteína foi considerada fator de risco independente para disfunção ventricular esquerda ou disfunção ventricular esquerda e falência cardíaca congestiva.

O valor preditivo das concentrações plasmáticas de homocisteína foi investigado no prognóstico da falência cardíaca congestiva, de origens isquêmica e não-isquêmica. Como em outros estudos, a média das concentrações plasmáticas de homocisteína foi maior estatisticamente no grupo de pacientes com falência coronariana congestiva $(15,80 \mu \mathrm{mol} / \mathrm{L})$ do que no grupo controle $(10,90 \mu \mathrm{mol} / \mathrm{L})$. Encontrou-se correlação positiva entre homocisteína, creatinina e idade. Entretanto, os pacientes com hiper-homocisteinemia apresentaram risco relativo de morte 4,23 vezes maior, sugerindo que a homocisteína é um forte fator preditor de mortalidade em pacientes com falência coronariana congestiva. Concentrações elevadas de homocisteína observadas em pacientes com falência cardíaca congestiva, qualquer que seja a etiologia da doença cardiovascular (isquêmica ou não isquêmica), foram correlacionadas à severidade da doença (49).

Desse modo, sugere-se que a hiper-homocisteinemia pode ser fator preditivo de doença cardiovascular e de mortalidade precoce em pacientes com a doença instalada. 


\section{HIPER-HOMOCISTEINEMIA E GENÉTICA}

Várias enzimas são envolvidas nas duas vias interconectadas do metabolismo da homocisteína, entre elas as enzimas C $\beta S$, MS e MTHFR. Variações nos genes que codificam essas enzimas podem ser importantes na determinação das concentrações plasmáticas de homocisteína em populações de alto risco para o desenvolvimento de doenças vasculares (18).

A mutação no gene que codifica a enzima $C \beta S$, que atua na via da transulfuração, é uma condição autossômica recessiva; nesse gene do cromossoma 21 (21q22.3), 33 mutações pontuais já foram descritas em diferentes populações, sendo que, em $50 \%$ dos casos, corresponderam às mutações G919A e T833C. Mutações CpG nos éxons 3, 6 e 7 podem causar hiper-homocisteinemia (6). Estudo caso-controle realizado com 95 pacientes com trombose venosa objetivou avaliar a frequência da mutação 844ins68 do gene da C $\beta S$. O genótipo heterozigoto para a mutação foi encontrado em $14,73 \%$ dos pacientes e em $3,1 \%$ dos indivíduos do grupo controle. A presença da mutação 844ins68 no gene da C $\beta S$ em heterozigose, com frequência aumentada entre portadores de trombose venosa profunda, sugere ser fator de risco para o desenvolvimento da doença (50). Estima-se que a frequência de mutação C $\beta S$ na população geral é de $1 \%$ e que $30 \%$ a $40 \%$ dos indivíduos com doença vascular precoce sejam heterozigotos para mutações da C $\beta S$ (51).

Podem ocorrer mutações do gene da MS, enzima dependente de vitamina B12, que catalisa a remetilação da homocisteína para metionina por desmetilação concorrente do 5-metiltetra-hidrofolato para tetrahidrofolato. Alterações estruturais e funcionais ocorrem quando há deficiência de cobalamina ou defeito na formação de metil-cobalamina, utilizada como cofator da enzima. Mutações do gene MS estão associadas à doença cardíaca isquêmica, principalmente em fumantes (6).

A enzima MTHFR tem um papel importante no metabolismo da homocisteína, pois ela catalisa a redução da 5,10-metilenotetrahidrofolato para 5-metiltetrahidrofolato, gerando, assim, a forma de folato necessária para a remetilação da homocisteína a metionina (52).

O gene humano que codifica a MTHFR é alvo de muitas investigações; o mapeamento da região cromossomal lp36.3, de aproximadamente $17 \mathrm{~Kb}$, encontrou 11 éxons distanciados por $2,2 \mathrm{~Kb}(52,53)$.

A deficiência grave da enzima MTHFR, um raro erro inato do metabolismo que ocorre na via da remetilação, é caracterizada por hiper-homocisteinemia e homocistinúria. Vinte e quatro raras mutações no gene da MTHFR foram associadas à deficiência grave da MTHFR, e indivíduos com estas mutações possuem atividade enzimática residual de $0 \%$ a $20 \%$, além de apresentar, na infância ou na adolescência, retardo mental, disfunção motora, distúrbios psiquiátricos, entre outras anormalidades neurológicas, e risco elevado para doenças vasculares (53).

Das várias mutações no gene da MTHFR que têm sido identificadas, algumas resultam na síntese de variantes termolábeis da MTHFR, ou seja, variantes que exibem atividade enzimática mínima após a desativação por calor a $46^{\circ} \mathrm{C}(54)$.

Em 1995, Frosst e cols. (53) identificaram a mutação C677T que converte o códon da alanina para o códon da valina, com a substituição de $\mathrm{C}$ para $\mathrm{T}$ no nucleotídeo 677 , e mostraram que essa mutação codifica a forma termolábil da MTHFR, previamente sugerida por Kang e cols. (54) como um fator de risco para a doença vascular.

A frequência de alelos varia com a população estudada, sendo que $8 \%$ da população geral tem genótipo homozigoto alterado para a variante termolábil e, em pacientes com doenças vasculares, observam-se cifras de $14 \%$ a 17\% (55). Existe grande influência das concentrações de ácido fólico em homozigotos e a hiper-homocisteinemia só ocorre quando os valores dessa vitamina são inferiores a 15,4 nmol/L. Por outro lado, a hiper-homocisteinemia ocorre em heterozigotos para essa mutação somente quando há deficiência de folato concomitante, e $15 \%$ dos pacientes com doença cardiovascular e apenas $5 \%$ dos controles apresentam a variante termolábil (25).

Uma nova mutação, no mesmo gene da MTHFR, foi notificada em 1998 por Van der Put e cols. (56). A mutação A1298C resulta na alteração do códon de glutamato para a alanina, conduzindo a diminuição na atividade da MTHFR, a qual é mais pronunciada em homozigotos mutantes do que em heterozigotos. A presença do alelo $\mathrm{C}$, entretanto, não foi associada à elevação de homocisteína plasmática ou à redução de ácido fólico no plasma, fenômenos evidentes na mutação C677T (56).

Estudo conduzido em pacientes com doença arterial coronariana sugere que ambos os genótipos, homozigoto mutante e heterozigoto para a mutação C677T, resultam na elevação moderada das concentrações plasmáticas de homocisteína, e que o genótipo homozigoto mutante é um modesto, mas significativo, fator de risco para a doença arterial coronariana (57). Em adição, metanálise com nove estudos envolvendo pacientes com doença arterial periférica mostrou forte associação en- 
tre o genótipo homozigoto mutante (677TT) e risco aumentado para essa doença (58).

Existem, entretanto, resultados contraditórios na literatura quanto à importância de mutações no gene da MTHFR como fatores de risco independentes para doenças vasculares. Polimorfismos no gene da MTHFR não foram associados ao espessamento da carótida em pacientes turcos com aterosclerose, mas concentrações de homocisteína encontravam-se elevadas (59). Metanálise com mais de 6.000 pacientes não mostrou relação significativa entre o polimorfismo C677T do gene que codifica a enzima MTHFR e doenças cardiovasculares (60).

Alguns autores sugerem que a homocisteína não é fator de risco para a doença vascular em razão de a mutação gênica - uma das principais causas de hiperhomocisteinemia - não estar significativamente associada a essa doença. Uma questão fundamental é saber se os argumentos, criticamente discutidos por Fletcher e Kessling (61), são válidos.

Se o risco do genótipo TT (mutação C677T) deriva do seu efeito sobre a homocisteína, o risco relativo esperado pode ser estimado a partir de dados publicados. Numa metanálise, Brättstrom e cols. (60) constataram que a concentração de homocisteína foi 2,6 $\mu \mathrm{mol} / \mathrm{L}$ maior naqueles com o genótipo TT do que naqueles com genótipo CC. Cálculos do tamanho amostral mostraram que, para detectar risco relativo da ordem de 1,10 a 1,15 , com um poder de $80 \%$ e nível de significância de $5 \%, 7.800$ a 16.300 casos e igual número de controles seriam necessários. Tal tamanho da amostra não foi alcançado em estudos que avaliaram a relação da MTHFR com doença cardiovascular. Assim, a falta de associação significativa entre a mutação C677T em heterozigose do gene que codifica a MTHFR não afasta a participação da homocisteína em doenças cardiovasculares. De fato, o risco relativo de 1,12 associado ao genótipo TT relatado por Brättstrom e cols. (60) concorda com o risco relativo esperado, com base em metanálise de estudos prospectivos conduzida por Ueland e cols. (62).

Outro assunto que tem gerado dúvidas é a grande diferença na força de associação entre o genótipo TT e doenças cardiovasculares encontrada quando se comparam vários estudos. Parte de tal diferença pode ser atribuída ao estado nutricional variável das populações, uma vez que indivíduos TT desenvolvem elevadas concentrações de homocisteína no plasma somente na condição de deficiência de ácido fólico (63). Em alguns estudos clínicos sobre a MTHFR, concentrações de folato e homocisteína não são determinadas (61). Em contraste, em estudo em homens portadores de doenças cardiovasculares, reduzidas concentrações de colesterol e ácido fólico, o genótipo TT foi preditivo da extensão da doença arterial coronariana (64).

Outro fator de confusão é a heterogeneidade genética dos casos comparados à população controle. A frequência do alelo $\mathrm{T}$ varia de nula, em negros americanos, a 16\%, em italianos (61). Desse modo, devido à interação genética ou nutricional, o polimorfismo C677T da MTHFR pode predizer risco para doença cardiovascular somente em certos grupos étnicos.

Recentemente, foi identificada uma nova mutação no éxon 11 do gene da MTHFR, a mutação G1793A, que resulta na substituição do aminoácido arginina por glutamina (65). Estudo conduzido com 83 indivíduos brasileiros portadores de diabetes melito tipo 2 mostrou que a frequência alélica para a mutação G1793A foi 3,01\%; portadores de alelos mutantes homozigóticos não foram detectados. Os autores ressaltam que indivíduos com genótipo heterozigoto para a mutação Gl793A mostraram valores limítrofes ou baixos de ácido fólico e de concentrações de vitamina Bl2, em comparação com os indivíduos com genótipo normal. Hiper-homocisteinemia e deficiência de vitaminas apresentadas por indivíduos diabéticos tipo 2, com genótipo heterozigoto para a mutação G1793A no gene MTHFR, atingiram valores normais pela suplementação diária com $1 \mathrm{mg}$ de ácido fólico/dia (66). Em estudo envolvendo 89 pacientes com insuficiência renal crônica em diálise, somente cinco deles $(5,62 \%)$ apresentaram a mutação G1793A e hiper-homocisteinemia e os autores concluíram não haver influência dos polimorfismos sobre as concentrações de homocisteína. Importante ressaltar que os pacientes, devido à doença renal, recebiam suplementação de rotina com vitaminas, incluindo o ácido fólico (67).

A descoberta da mutação G1793A e de novas mutações nos genes da MTHFR, C $\beta S$ e MS são de grande importância clínica. Investigações adicionais sobre a frequência dessas mutações em diferentes populações e sobre a interação desses polimorfismos com mutações já descritas, com o metabolismo do ácido fólico e com o desenvolvimento da hiper-homocisteinemia, podem auxiliar no melhor entendimento do complexo sistema que envolve o metabolismo do ácido fólico.

\section{HIPER-HOMOCISTEINEMIA E VITAMINAS DO COMPLEXO B}

Os efeitos da nutrição (vitaminas do complexo B), medicamentos (metotrexato e anticonvulsivantes), con- 
dições patológicas (doença renal, hipotireoidismo) e outras doenças sobre as concentrações plasmáticas de homocisteína têm sido bem documentados (36).

Algumas vitaminas funcionam como cofatores e substratos no metabolismo da metionina e homocisteína. O ácido fólico e a cianocobalamina (B12) regulam a via metabólica catalisada pela enzima MTHFR e MS, respectivamente, enquanto a piridoxina (B6) é um cofator da C $\beta S$. Um grande número de estudos tem demonstrado correlação inversa entre as concentrações plasmáticas ou séricas de homocisteína com ácido fólico, vitamina B12 e vitamina B6 $(27,36,43,66)$.

$\mathrm{O}$ estado nutricional, isolado ou em associação com alguns fatores genéticos, assim como a MTHFR termolábil, causa várias formas de hiper-homocisteinemia $(20,35)$. Para o tratamento da hiper-homocisteinemia é importante identificar a base de sua etiologia e a severidade do(s) defeito(s) genético(s). O método preferido para o tratamento da hiper-homocisteinemia genética é a ativação da atividade da enzima mutante com o cofator ou o precursor do cofator. Essa prática não corrige a hiper-homocisteinemia, mas a suplementação com doses farmacológicas de betaína ou de ácido fólico poderiam ser utilizadas como alternativa para melhorar o turnover da homocisteína (19).

Na presença da mutação da MTHFR termolábil, quantidades de ácido fólico e vitamina B12, acima das recomendações nutricionais (69), têm sido sugeridas para regular as concentrações de homocisteína e potencialmente reduzir o risco de doenças vasculares $(22,66)$.

Desse modo, a suplementação com ácido fólico, vitamina B12 e, em alguns casos, com a vitamina B6, acima das concentrações séricas de normalidade poderia ser efetiva na redução da hiper-homocisteinemia moderada (19). Entretanto, o ácido fólico, a vitamina Bl2 e a vitamina B6 diferem quanto ao seu potencial para influenciar as concentrações plasmáticas de homocisteína. A vitamina B6, quando oferecida isoladamente, não tem apresentado efeito na redução das concentrações de homocisteína (42). Em indivíduos do sexo masculino com concentrações elevadas de homocisteína, a suplementação com vitamina B12 (acima de $400 \mu \mathrm{g} /$ dia) resultou em diminuição de $15 \%$ nas concentrações iniciais de homocisteína. Ressalta-se que, entre as vitaminas citadas, o ácido fólico apresenta papel fundamental na redução das concentrações plasmáticas de homocisteína. A suplementação com ácido fólico isolado, na quantidade de $0,65 \mathrm{mg} / \mathrm{dia}$, promoveu redução de $42 \%$ nas concentrações plasmáticas de homocisteína (68).
A ingestão média diária recomendada de ácido fólico e vitamina B12 para indivíduos saudáveis, de ambos os sexos, de 14 a > 70 anos, é de $400 \mu \mathrm{g} /$ dia e 2,4 $\mu \mathrm{g}$ / dia, respectivamente (69).

A suplementação isolada com ácido fólico, nas doses de $0,2 \mathrm{a} 15 \mathrm{mg} /$ dia, pode diminuir as concentrações de homocisteína plasmática sem toxicidade aparente (60).

Em metanálise ( $\mathrm{n}=20.669$ indivíduos), sugeriu-se que a redução de $3 \mu \mathrm{mol} / \mathrm{L}$ nas concentrações basais de homocisteína, por meio da suplementação com ácido fólico na quantidade de $0,8 \mathrm{mg} /$ dia, poderia reduzir o risco de doença isquêmica do coração em 16\% (11\% a $20 \%)$, trombose venosa em $25 \%$ ( $8 \%$ a $38 \%$ ) e derrame em $24 \%$ ( $15 \%$ a $33 \%)(46)$.

Estudos com suplementação de ácido fólico em doses de $0,5 \mathrm{a} 2 \mathrm{mg} / \mathrm{dia}$, por um período de três a quatro semanas, evidenciam que maiores reduções nas concentrações plasmáticas de homocisteína são observadas em indivíduos com genótipo homozigoto mutante para o polimorfismo C677T quando comparados aos indivíduos homozigotos normais ou heterozigotos $(22,70)$.

A ingestão adequada de ácido fólico é sugerida para indivíduos com mutação no gene que codifica a MTHFR, mesmo na ausência da deficiência clínica dessa vitamina, com a finalidade de regular as concentrações plasmáticas de homocisteína e, potencialmente, reduzir o risco para doenças vasculares.

A frequência da suplementação com ácido fólico pode influenciar as concentrações de homocisteína. A suplementação diária com ácido fólico na quantidade de $250 \mu$ foi mais efetiva na redução das concentrações plasmáticas de homocisteína do que uma dose dupla $(500 \mu \mathrm{g})$ oferecida em dias alternados (71). O Food and Nutrition Board do Institute of Medicine (69) tem recomendado limite máximo de $1 \mathrm{mg} / \mathrm{dia}$ de ácido fólico, sugerindo que doses maiores poderiam mascarar sinais de deficiência de vitamina $\mathrm{Bl} 2 \mathrm{em}$ alguns indivíduos.

Hiper-homocisteinemia foi diagnosticada em 27,7\% dos pacientes com diabetes melito tipo 2 , deficiência de ácido fólico em 15,6\%, e, de vitamina B12, deficiência de 7,23\%. Homocisteína plasmática foi inversamente correlacionada às concentrações de ácido fólico $(\mathrm{r}=-0,27, \mathrm{p}=0,01)$ e de vitamina $\mathrm{B} 12(\mathrm{r}=-0,21$, $\mathrm{p}=0,05)$. A hiper-homocisteinemia foi corrigida na totalidade dos indivíduos com diferentes genótipos para as mutações C677T, Al298C e G1793A do gene da MTHFR após três meses de suplementação com $1 \mathrm{mg}$ de ácido fólico/dia (22). 
O efeito do ácido fólico na redução das concentrações de homocisteína pode ser explicado bioquimicamente. No metabolismo da homocisteína, as vitaminas B6 e B12 atuam como coenzimas e, assim, não são utilizadas em excesso durante a reação na qual elas estão envolvidas. O ácido fólico, entretanto, funciona como um doador do grupo metil na reação de remetilação e é utilizado em quantidades ascendentes até que seja convertido em 5-metiltetra-hidrofolato. Durante a reação de remetilação, o grupo metil do 5-metiltetra-hidrofolato é transferido para a homocisteína pela vitamina B12 para, posteriormente, formar a metionina. Por essa razão, o ácido fólico age como fator limitante para tal reação, e a ausência desse doador do grupo metil não pode ser compensada pela vitamina B12. Esta é estocada em quantidades suficientes no organismo, e sua deficiência não é habitual - exceto em indivíduos com deficiência de fator intrínseco e em vegetarianos. Dessa forma, a vitamina B12 tem sido considerada menor determinante das concentrações plasmáticas de homocisteína do que o ácido fólico (72).

Por outro lado, em indivíduos com concentrações de vitamina B12 abaixo da normalidade, a resposta máxima à suplementação de ácido fólico não pode ser alcançada com êxito, a menos que a vitamina B12 seja oferecida concomitantemente (73). Indivíduos homozigotos para a mutação C677T do gene da MTHFR exibiram menores concentrações plasmáticas de vitamina B12, quando comparados aos indivíduos com demais genótipos (74).

A relação causal aparente entre genótipo e concentrações plasmáticas de ácido fólico indica que, em indivíduos com concentrações plasmáticas elevadas de homocisteína, as concentrações de ácido fólico reduzidas não são atribuídas necessariamente à ingestão dietética insuficiente dessa vitamina, mas, ao menos em parte, deve ser resultado direto da atividade reduzida da MTHFR termolábil.

Dessa forma, o tratamento da hiper-homocisteinemia varia de acordo com a causa subjacente. A normalização das concentrações plasmáticas de homocisteína ocorre, em média, dentro de quatro a seis semanas após o início do tratamento. Sugere-se que a suplementação não ultrapasse o limite máximo tolerável das vitaminas sugerido pelas Dietary Reference Intakes (DRI's) do Food and Nutrition Board do Institute of Medicine (69). O tratamento é barato e seguro, porém, as doses mínimas efetivas de ácido fólico e vitaminas B12 e B6 ainda não foram estabelecidas.

\section{CONCLUSÕES}

A associação entre hiper-homocisteinemia e doenças vasculares tem sido consistentemente demonstrada, sendo a homocisteína apontada como fator de risco independente. Concentrações elevadas de homocisteína inibem os mecanismos anticoagulantes do endotélio vascular e aumentam a formação de radicais livres favorecendo eventos vasculares.

As várias formas de hiper-homocisteinemia são causadas por defeitos genéticos ou não genéticos, ou pela combinação de ambos os fatores. Quanto aos defeitos genéticos, mutações em enzimas chaves do metabolismo da homocisteína foram descritas, sendo que muitos indivíduos apresentam maiores concentrações plasmáticas de homocisteína e menores concentrações de vitaminas do complexo B (ácido fólico, B12 e B6).

$\mathrm{O}$ estado nutricional deficiente em ácido fólico, B12 e B6 (defeitos não genéticos) é a causa mais comum da hiper-homocisteinemia moderada. Desse modo, estimular o consumo de alimentos fontes dessas vitaminas do complexo B constitui um método simples, eficaz e econômico na prevenção da hiper-homocisteinemia, podendo contribuir para a redução dos riscos de doenças vasculares. Outra forma terapêutica possível é fundamentada na suplementação com cápsulas ou comprimidos contendo ácido fólico isolado ou em associação com vitaminas B12 e B6.

A complexa interação entre polimorfismos genéticos e deficiências de vitaminas do complexo B ainda é foco de extensa investigação. O conhecimento do mapa de predisposições genéticas de cada indivíduo permitirá ajustar seu estilo de vida ao seu genoma e, assim, reduzir o risco de doenças - entre elas, as vasculares. Para tanto, há ainda necessidade de realização de estudos randomizados e controlados, envolvendo maiores casuísticas.

Declaração: os autores declaram não haver conflitos de interesse científico neste estudo.

\section{REFERÊNCIAS}

1. Brasil. Ministério da Saúde. Taxa de mortalidade específica por doenças do aparelho circulatório. 2005. Disponível em: http://tabnet.datasus.gov.br/cgi/deftohtm.exe?idb2007/c08.def Acesso em: 13 fev. 2009.

2. Santos Filho RD, MartinezTLR. Fatores de risco para doença cardiovascular: velhos e novos fatores de risco, velhos problemas! Arq Bras Endocrinol Metab. 2002; 46(3):212-4.

3. Neves LB, Macedo DM, Lopes AC. Homocisteína. J Bras Patol Med Lab. 2004;40(5):311-20. 
4. Nair KG, AshavaidTF, Nair SR, Eghlim FF. The genetic basis of hiperhomocysteinemia. IHJ. 2000;52(7 Suppl)S16-7.

5. Muniz MTC, Siqueira ERF, Fonseca RA, Dalmeida V, Hotta JK, dos Santos JE, et al. Avaliação da relação entre o polimorfismo C677T no gene para MTHFR e a concentração plasmática de homocisteína na doença arterial coronariana. Arq Bras Endocrinol Metab. 2006;50(6):1059-65.

6. May HT, Alharenthi R, Anderson JL, Muhlestein JB, Reyna SP, Bair $\mathrm{TL}$, et al. Homocysteine levels are associated with increased risk of congestive heart failure in patients with and without coronary artery disease. Cardiology. 2007;107(3):178-84.

7. Graham IM, Daly LE, Refsum HM, Robinson K, Brattstrom LE, Ueland PM, et al. Plasma homocysteine as a risk factor for cardiovascular disease:The European Concerted Action Project. JAMA. 1997;277(22):1775-81.

8. Wolfgang $\mathrm{H}$, Obeid $\mathrm{R}$, Jouma M. Hyperhomocysteinemia and vitamin B-12 deficiency are more striking in Syrians than in Germans - causes and implications. Atherosclerosis. 2003;166(1):143-50.

9. Mager A, Lalezari S, Shohat T, Birnbaum Y, AdlerY, Magal N, et al. Methylenetetrahydrofolate reductase genotypes and early-onset coronary artery disease. Circulation. 1999;100(24):2406-10.

10. Tsai MY, Welge BG, Hanson NQ, Bignell MK, Vessey J, Schwichtenberg K, et al. Genetic causes of mild hyperhomocysteinemia in patients with premature occlusive coronary artery diseases. Atherosclerosis. 1999;143(1):163-70.

11. Chao CL, Tsai HH, Lee CM, Hsu SM, Kao JT, Chien KL, et al.The graded effect of hyperhomocysteinemia on the severity and extent of coronary atherosclerosis. Atherosclerosis. 1999;147(2):379-80.

12. Yoo JH, Park JE, Hong KP, Lee SH, Kim DK, Lee WR, et al. Moderate hyperhomocysteinemia is associated with the presence of coronary artery disease and the severity of coronary atherosclerosis in Koreans. Thromb Res. 1999;94(1):45-52.

13. Anderson JL, Muhlesteim JB, Horne BD, Carlquist JF, BairTL, Madsen TE, et al. Plasma homocysteine predicts mortality independently of traditional risk factors and C-reactive protein in patients with angiographically defined coronary artery disease. Circulation. 2000;102(11):1227-32.

14. Hodis HN, Mack WJ, Dustin L, Mahrer PR, Azen SP, Detrano R, Selhub J, Alaupovic P, Liu CR, Liu CH, Hwang J, Wilcox AG, Selzer RH; BVAIT Research Group. High-dose B vitamin supplementation and progression of subclinical atherosclerosis. A randomized controlled trial. Stroke. 2009;40(3):730-6.

15. Potter K, Hankey GJ, Green DJ, Eikelboom J, Jamrozik K, Arnolda LF. The effect of long-term homocysteine-lowering on carotid intima-media thickness and flow-mediated vasodilation in stroke patients: a randomized controlled trial and meta-analysis. BMC Cardiovasc Disord. 2008;20:8-24

16. Andreassi MG, Botto N, Cocci F, Battaglia D, Antonioli E, Masetti $\mathrm{S}$, et al. Methylenetetrahydrofolate reductase gene C677T polymorphism, homocysteine, vitamin B12, and DNA damage in coronary artery disease. Hum Genet. 2003;112(2):171-7.

17. Saw SM, Yuan JM, Ong CN, Arakawa K, Lee HP, Coetzee GA. Genetic, dietary and other lifestyle determinants of plasma homocysteine concentrations in middle-aged and older Chinese men and women in Singapore. Am J Clin Nutr. 2001;73(2):232-9.

18. Dekou V, Whincup P, Papacosta O, Ebrahim S, Lennon L, Ueland $\mathrm{PM}$, et al. The effect of the C677T and $\mathrm{A} 1298 \mathrm{C}$ polymorphisms in the methylenetetrahydrofolate reductase gene on homocysteine levels in elderly men and women from the British regional heart study. Atherosclerosis. 2001;154(3):659-66.

19. Kang SS. Treatment of hyperhomocyst(e)inemia: physiological basis. J Nutr. 1996;126(Suppl 4):1273-5.

20. Bailey LB, Gregory JF 3rd. Polymorphisms of methylenetetrahydrofolate reductase and other enzymes: metabolic significance, risks and impact on folate requeriment. J Nutr. 1999;129(5):919-22.
21. Lewis SJ, Ebrahim S, Smith GD. Meta-analysis of MTHFR 677 C->T polymorphism and coronary heart disease: does totality of evidence support causal role for homocysteine and preventive potential of folate? BMJ. 2005;331(7524):1053-9.

22. Melo SS. Efeito da suplementação com ácido fólico sobre as concentrações de homocisteína em diferentes genótipos da metilenotetra-hidrofolato redutase em pacientes diabéticos tipo 2 [tese]. São Paulo: Universidade de São Paulo; 2003.

23. Refsum $H$, Ueland PM, Nygard O, Vollset SE. Homocysteine and cardiovascular disease. Annu Rev Med. 1998;49(1):31-62.

24. Eikelboom JW, Lonn E, Genest J Jr, Hankey G, Yusuf S. Homocyst(e)ine and cardiovascular disease: a critical review of the epidemiologic evidence. Ann Intern Med. 1999;131(5):363-75.

25. Fonseca V, Guba SC, Fink LM. Hyperhomocysteinemia and endocrine system: implications for atherosclerosis and thrombosis. Endocr Rev. 1999;20(5):738-59.

26. AubardY, Darodes N, Cantaloube M. Hyperhomocysteinemia and pregnancy - review o four present understanding and therapeutic implications. Eur J Obst Gynec Reprod Biol. 2000;93(2):157-65.

27. Selhub J. Homocysteine metabolism. Annu Rev Nutr. 1999; 19(1):217-46.

28. Saw SM, Yuan JM, Ong CN, Arakawa K, Lee HP, Coetzee GA. Genetic, dietary and other lifestyle determinants of plasma homocysteine concentrations in middle-aged and other Chinese men and women in Singapore. Am J Clin Nutr. 2001;73(2):232-9.

29. De Vigneaud VE. Trail of research in sulfur chemistry and metabolism, and related fields. Ithaca, N.Y: Cornell University Press, 1952. In: Carson NAJ, Neil DW. Metabolic abnormalities detected in a survey of mentally backward individuals in Northern Ireland. Arch Dis Child. 1962;37: 505-13.

30. Carson NAJ, Neil DW. Metabolic abnormalities detected in a survey of mentally backward individuals in Northern Ireland. Arch Dis Child. 1962;37:505-13.

31. Geritsen T, Waisman HA. Homocystinuria, an error in the metabolism of methionine. Pediatrics. 1964;33:413-20.

32. McCully KS. Vascular pathology of homocysteinemia: implications for the pathogenesis of arteriosclerosis. Am J Pathol. 1969;56(1):111-28.

33. Gupta VJ, Wilcken DEL. The detection of cysteine-homocysteine mixed disulphide in plasma of normal fasting man. Eur $\mathrm{J}$ Clin Invest. 1978;8(4):205-7.

34. Boers GH, Smals AG, Trijbels FJ, Fowler B, Bakkeren JA, Schoonderwaldt $\mathrm{HC}$, et al. Heterozygosity for homocystinuria in premature peripheral and cerebral occlusive arterial disease. N Engl J Med. 1985;313(12):709-15.

35. Kang SS, Wong PWK, Malinow, MR. Hyperhomocisteinemia as a risk factor for occlusive vascular disease. Annu Rev Nutr. 1992;12:279-98.

36. Ueland PM, Refsum H. Plasma homocysteine, a risk factor for vascular disease, and drug therapy. J Lab Clin Med. 1989;114(5):473-501.

37. Harboe-Gonçalves L, Vaz LS, Buzzi M. Avaliação dos níveis de hiper-homocisteinemia, vitamina $E$, selênio, cobre, ceruloplasmina e ferritina em pacientes com diagnóstico de acidente vascular cerebral isquêmico. J Bras Patol Med Lab. 2007;43(1):9-15.

38. Durand P, Prost M, Loreau N, Lussier-Cacan S, Blache D. Impaired homocysteine metabolism and atherothrombotic disease. Lab Invest. 2001;81(5):645-72.

39. Eberhardt RT, Forgione MA, Cap A, Leopold JA, Rudd MA, Trolliet $\mathrm{M}$, et al. Endothelial dysfunction in a murine model of mild hyperhomocyst(e)inemia. J Clin Invest. 2000;106(4):483-91.

40. Okada E, Oida K, Tada H, Asazuma K, Eguchi K, Tohda G, et al. Hyperhomocysteinemia is a risk factor for coronary arteriosclerosis in japanese patients with type 2 diabetes. Diabetes Care. 1999;22(3):484-90.

41. Crott JW, Fenech M. Preliminary study of the genotoxic potencial of homocysteine in human lymphocytes in vitro. Mutagenesis. 2001:16(3):213-7. 
42. Boushey CJ, Beresford AS, Omenn GS, Motulsky AG. A quantitative assessment of plasma homocysteine as arisk factor for vascular disease. Probable benefits of increasing folic acid intakes. JAMA. 1995;274(13):1049-57.

43. Malinow MR, Duell PB, Hess DL, Anderson PH, Kruger WD, Phillipson $\mathrm{BE}$, et al. Reduction of plasma homocysteine levels by breakfast cereal fortified with folic acid in patients with coronary heart disease. N Engl J Med. 1998;338(15):1009-15.

44. Nygard $\mathrm{O}$, Nordrehaug JE, Refsum $\mathrm{H}$, Ueland PM, Farstad M, Vollset SE. Plasma homocysteine levels and mortality in patients with coronary artery disease. N Engl J Med. 1997;337(4):230-6.

45. Uehara SK, Baluz K, Rosa G. Possíveis mecanismos trombogênicos da hiper-homocisteinemia e o seu tratamento nutricional. Rev Nutr. 2005;18(6):743-51.

46. Wald DS, Law M, Morris JK. Homocysteine and cardiovascular disease: evidence on causality from a meta-analysis. BMJ. 2002;325(7374):1-7.

47. Franken DG, Boers GH, Blom HJ, Trijbels JM. Effect of various regimens of vitamin $\mathrm{B}_{6}$ and folic acid on mild hyperhomocysteinemia in vascular patients. J Inherited Met Dis. 1994;17(1):159-62.

48. May HT, Alharenthi R, Anderson JI, Muhlestein JB, Reyna SP, Bair $\mathrm{Tl}$, et al. Homocysteine levels are associated with increased risk of congestive heart failure in patients with and without coronary artery disease. Cardiology. 2007;107(3):178-84.

49. Gibelin P, Serre S, Candito M, Houcher B, Berthier F, Baudouy M. Prognostic value of homocysteinemia in patients with congestive heart failure. Clin Chem Lab Med. 2006;44(7):813-6.

50. Bonini-Domingos CR, Zamaro PJA, Mendiburu CF, Sanches FE, Cintra JR, Godoy JMP, et al. Freqüência da mutação 844ins68 do gene da cistationina $\beta$-sintetase em pacientes com trombose venosa profunda. Rev Bras Hematol Hemoter. 2005;27(1):12-5.

51. Tsai MY, Hanson NO, Schwichtenberg K, Garg U. Amplification refractory mutation system to identify mutations in cystationine $\beta$-synthase deficiency. Clin Chem 1995;41(12 Pt 1):1775-7.

52. Rosenberg N, Murata M, IkedaY, Opare-Sem O, Zivelin A, Geffen E, et al.The frequent 5,10-methylenetetrahydrofolate reductase C677T polymorphism is associated with a comumm haplotype in whites, Japanese and Africans. Am J Hum Genet. 2002;70(3):758-62.

53. Frosst $P$, Blom HJ, Milos R, Goyette $P$, Sheppard CA, Matthews $\mathrm{RG}$, et al. A candidate genetic risk factor for vascular disease: a common mutation in methylenetetrahydrofolate reductase. Nat Genet. 1995;10(1):111-3.

54. Kang SS, Zhou J, Wong PWK, Kowalisym J, Strokosch G. Intermediate homocysteinemia: a thermolabile variant of methylenetetrahydrofolate reductase. Am J Hum Genet. 1988;43(4):414-21.

55. Jacques PF, Bostom AG, Williams RR, Ellison RC, Eckfeldt JH, Rosenberg IH, et al. Relation between folate status, a common mutation in methylenetetrahydrofolate reductase, and plasma homocysteine concentrations. Circulation. 1996;93(1):7-9.

56. Van der Put NM, Steegers-Theunissen RP, Frosst P, Trijbels FJ, Eskes TK, Van der Heuvel LP, et al. Molecular genetic analysis in mild hyperhomocysteinemia: a common mutation in the methylenetetrahydrofolate reductase gene is a genetic factor for cardiovascular disease. Am J Hum Genet. 1996;58(1):35-41.

57. Kluijtmans LAJ, Kastelein JJP, Lindemans J, Boers GHJ, Heil SG, Bruschke AVG, et al. Thermolabile methylenetetrahydrofolate reductase in coronary artery disease. Circulation. 1997;96(8):2573-7.

58. Khandanpour N, Willis G, Meyer FJ, Armon MP, Loke YK, Wright AJ, et al. Peripheral arterial disease and methylenetetrahydrofolate reductase (MTHFR) C677T mutations: a case-control study and meta-analysis. J Vasc Surg. 2009;49(3):711-8.

59. Alioglu E, Turk U, Cam S, Abbasaliyev A, Tengiz I, Ercan E. Polymorphism of the methylenetetrahydrofolate reductase, vascular endothelial growth factor, endothelial nitric oxide synthase, monocyte chemoattractant protein-1 and apolipoprotein E genes are not associated with carotid intima-media thickness. Can J Cardiol. 2009;25(1):1-5.

60. Brättstrom L, Wilcken DE, Ohrvik J, Brudin L. Common methylenetetrahydrofolate reductase gene mutation leads to hyperhomocysteinemia but not to vascular disease: the results of a metaanalysis. Circulation. 1998;98(23):2520-6.

61. Fletcher $\mathrm{O}$, Kessling AM. MTHFR association with arteriosclerotic vascular disease? Hum Genet. 1998;103(1):11-21.

62. Ueland PM, Refsum H, Beresford SAA, Vollset SE. The controversy over homocisteine and cardiovascular risk. Am J Clin Nutr. 2000;72(2):324-32.

63. Girelli D, Friso S, Trabetti E, Olivieri O, Russo C, Pessotto R, et al. Methylenetetrahydrofolate reductase C677T mutation, plasma homocysteine, and folate in subjects from northem Italy with or without angiographically documented severe coronary atherosclerotic disease: evidence for an important genetic environmental interaction. Blood. 1998;91(11):4158-63.

64. Tokgözoğlu SL, Alikaşifoğlu M, Unsal, Atalar E, Aytemir K, Ozer N, et al. Methylene tetrahydrofolate reductase genotype and the risk and extent of coronary artery disease in a population with low plasma folate. Heart. 1999;81(5):518-22.

65. Rady PL, Szucs S, Grady J, Hudnall SD, Kellner LH, Nitowsky H, et al. Genetic polymorphisms of methylenetetrahidofolate reductase (MTHFR) and methionine syntase reductase (MTRR) in ethnic populations in Texas; a report of a novel MTHFR polymorphic site, G1793A. Am J Med Genet. 2002;107(2):162-8.

66. Melo SS, Persuhn DC, Meirelles MS, Jordao AA, Vannucchi H. G1793A polymorphisms in the methylene-tetrahydrofolate gene: effect of folic acid on homocysteine levels. Mol Nutr Food Res. 2006;50(8):769-74.

67. Domenici FA, Vannucchi MT, Simões-Ambrósio LM, Vannucchi $\mathrm{H}$. Hyperhomocysteinemia and polymorphisms of the methylenetetrahydrofolate gene in hemodialysis and peritoneal dialysis patients. Mol Nutr Food Res. 2007;51(11):1430-6.

68. Ubbink JB, Vermaak WJ, Van der Merwe A, Becker PJ, Delport R, Potgieter HC. Vitamin requeriment for the treatment of hyperhomocysteinemia in humans. J Nutr. 1994;124(10):1927-33.

69. Food and Nutrition Board - Institute of Medicine. Dietary Reference Intakes for thiamin, riboflavin, niacin, vitamin B6, vitamin B12, pantothenic acid, biotin, and choline: a report by the Standing Committee on the Scientific Evaluation of Dietary Reference Intakes and its panel on folate, other B vitamins and choline. National Academy Press, Washington, DC 2000. Disponível em: http://www.nap.edu/ catalog.php?record_id=6015\#toc. Acesso em: 12 fev. 2009

70. Malinow MR, Nieto FJ, Kruger WD, Duell PB, Hess DL, Gluckman $R A$, et al.The effects of folic acid supplementation on plasma total homocysteine are modulated by multivitamin use and methylenetetrahydrofolate reductase genotypes. Arterioscler Thromb Vasc Biol. 1997;17(6):1157-62.

71. Brouwer IA, Van Dusseldorp M, Thomas CMG, Duran M, Hautvast JGAJ, Eskes TKAB, et al. Low dose folic acid supplementation drecreases plasma homocysteine concentrations: a randomized trail. Indian Heart J. 2000;52(Suppl7):53-8.

72. Quinlivan EP, Mcpartlin J, Mcnulty H, Ward M, Strain JJ, Weir DG, et al. Importance of both folic acid and vitamin B12 in reduction of risk of vascular disease. Lancet. 2002;359(9302):227-8.

73. Landgren $F$, Israelsson $B$, Lindgren $A$, Hultberg $B$, Andersson $A$, Brattstrom L. Plasma homocysteine in acute myocardial infarction: homocysteine-lowering effect of folic acid. J Intern Med. 1995;237(4):381-8.

74. D'Angelo A, Coppola A, Madonna P, Fermo I, Pagano A, Mazzola $G$, et al. The role of vitamin $B_{12}$ in fasting hyperhomocysteinemia and its interaction with the homozygous C677T mutation of the methylenetetrahydrofolate reductase (MTHFR) gene: a case-control study of patients with early-onset thrombotic events. Thromb Haemost. 2000;83(4):563-70. 